\title{
Substantial reduction in the number of amputations among patients with diabetes: a cohort study over 16 years
}

\author{
Benjamin S. B. Rasmussen ${ }^{1}$ (1) - Knud B. Yderstraede ${ }^{1} \cdot$ Bendix Carstensen $^{2}$ • \\ Ole Skov $^{3} \cdot$ Henning Beck-Nielsen ${ }^{1}$
}

Received: 4 June 2015 / Accepted: 11 September 2015 / Published online: 22 November 2015

(C) Springer-Verlag Berlin Heidelberg 2015

\begin{abstract}
Aims/hypothesis The aim of this study was to describe the trends in rates of amputation among individuals with and without diabetes.

Methods We studied amputation rates in the County of Funen (approximately 0.5 million residents) during the period 1996-2011. Amputations were identified from the hospital administrative system, diabetes status by linkage with the Danish National Diabetes Register, and mortality and population data by extraction from Statistics Denmark. Amputation rates were analysed using proportional hazard models. We analysed the incidence of the first amputation at each level as well as the incidence of further amputations, subdivided by level of amputation.

Results During the period 1996-2011, a total of 2,832 amputations were performed, of which 1,285 were among patients with diabetes and 1,547 among individuals without diabetes. Relative to persons without diabetes, patients with diabetes had an HR for below-ankle amputations (BAAs) of 14.7 for men and 7.5 for women, and for from-ankle-to-knee amputations (BKAs) of 7.6 and 8.4 for men and women, respectively. For above-knee amputations (AKAs) the numbers were
\end{abstract}

Electronic supplementary material The online version of this article (doi:10.1007/s00125-015-3781-7) contains peer-reviewed but unedited supplementary material, which is available to authorised users.

Benjamin S. B. Rasmussen

bsr@rsyd.dk

1 Department of Medical Endocrinology, Odense University Hospital Denmark, Sdr. Boulevard 29, 5000 Odense C, Denmark

2 Clinical Epidemiology, Steno Diabetes Centre, Gentofte, Denmark

3 Department of Orthopaedic Surgery, Odense University Hospital, Odense, Denmark
4.0 for men and 3.7 for women. We found an annual reduction in BAA rates among patients with diabetes of $9.8 \%$, and the annual reduction in BKA for patients with diabetes was $15.1 \%$.

Conclusions/interpretation The amputation rate in patients with diabetes is still several-fold higher than in persons without diabetes, but the improvements in diabetes care in recent years have resulted in a steady decline in amputation rates among patients with diabetes from this Danish cohort.

Keywords Amputation · Diabetes · Diabetic patients

$\begin{array}{ll}\text { Abbreviations } \\ \text { AKA } & \text { Above-knee amputation } \\ \text { BAA } & \text { Below-ankle amputation } \\ \text { BKA } & \text { From-ankle-to-knee amputation } \\ \text { CRS } & \text { Civil Registration System } \\ \text { DFU } & \text { Diabetic foot ulcer } \\ \text { NDR } & \text { Danish National Diabetes Register } \\ \text { NHSR } & \text { National Health Services Register } \\ \text { PPV } & \text { Positive predictive value } \\ \text { RMPS } & \text { Register of Medicines Products Statistics }\end{array}$

Introduction

Individuals suffering from diabetes mellitus have an increased risk of developing cardiovascular disease. In addition, complications such as peripheral neuropathy combined with cardiovascular disease frequently lead to a diabetic foot ulcer (DFU). The presence of an ulcer is associated with higher all-cause mortality [1], and diabetes is a major contributor to non-traumatic amputation. Among amputees, $85 \%$ will initially present with a DFU [2]. The cost of amputations is high [3], 
and the total costs are subject to a number of confounders and are thus difficult to estimate precisely. In addition, a recent study has shown a 30 day mortality of $30 \%$ after a major amputation and more than 50\% mortality 1 year after amputation [4].

Reductions in amputation rate among patients with diabetes have been reported on several occasions, although there is a variation between different settings [5-9]. In individuals presenting with a DFU, it is likely that the overall reduction in amputation rate is related to a more aggressive vascular surgical approach [10, 11], although this is somewhat disputed [12]. DFUs are usually handled in a multidisciplinary setting involving different surgical and medical specialties [13]. It seems that a multidisciplinary approach to patients with diabetes can provide a further reduction in the rate of amputation [14]. The registration of amputations among patients with diabetes is substantially affected by differences in registration modes and data collection [8], and a significant difference in amputation rate could also reflect differences in local healthcare delivery, as suggested by Holman et al [5].

In Denmark, however, the linkage of national registers, local hospital databases and amputation databases renders assessment very reliable. Based on these reliable databases, the aim of this study was to calculate the amputation rates in both patients with diabetes and non-diabetic individuals in cohorts from the County of Funen over a period of 16 years (1996-2011).

\section{Methods}

\section{Design}

All individuals in the County of Funen (approx. 0.5 million inhabitants), Denmark, were followed for occurrences of amputation, diagnosis of diabetes and death during the period 1996-2011, with the aim of describing amputation rates and comparing these between individuals with and without diabetes.

\section{Data}

Amputation data We identified all individuals with a record of amputation from the administrative system at Odense University Hospital. Amputation data were predefined by surgical codes categorising amputation level. Each contact was recorded with one or more diagnoses and procedure codes according to the Nordic Classification of Surgical Procedures [15] and the ICD-10 system (www.who.int/classifications/icd/en/); “*” was used as a wildcard including all relevant subcodes. Amputations were categorised as follows:

- below-ankle amputation (BAAs): ankle, foot and toe, KNHQ0*, KNHQ1* or KNHQ9*
- from-ankle-to-knee amputations (BKAs): knee and lower leg, KNGQ0*, KNGQ1* or KNGQ9*

- above-knee amputations (AKAs): hip and thigh, $\mathrm{KNFQ} 0 *$ KNFQ1* or KNFQ9*

We defined amputation status as the highest located amputation performed at any time. Thus, a BAA on the right foot in a person already having had a BKA on the left leg was not considered a BAA event. A person could contribute to all three amputation categories, but only in increasing sequence over time (BAA $\rightarrow$ BKA $\rightarrow$ AKA), and only by the first amputation in each category.

We noted a group of very young individuals who were registered as amputees. After a thorough investigation, most of these amputations were shown to have been performed because of congenital malformations (e.g. extra toes), and all individuals below the age of 15 were therefore excluded from the follow-up analyses.

Diabetes data We collected the following variables from the Danish National Diabetes Register (NDR) [16]: ID, sex, date of birth, date of inclusion in the NDR (corresponding to the date of diagnosis of diabetes) and date of death. The NDR comprises persons meeting one of the following criteria, the inclusion date being defined as the date on which the first criterion was met.

1. A diagnosis of diabetes in the National Patient Register defined as ICD-10 (www.who.int/classifications/icd/en/) DE10-14, DH36.0 or DO24 (excluding DO24.4), and ICD-8 (prior to 1994).

2. Registration of chiropody in a diabetic patient in the National Health Services Register (NHSR).

3. Five blood glucose measurements during a 1 year period in the NHSR.

4. Two blood glucose measurements per year in five consecutive years in the NHSR.

5. The second purchase of oral glucose-lowering drugs in the prescription register, the Register of Medicines Products Statistics (RMPS), with the exception of women aged 20-39 purchasing metformin only, because metformin is also used as a medication for polycystic ovarian syndrome.

6. The second purchase of prescribed insulin recorded in the RMPS [16].

The database is estimated to have a sensitivity of $\geq 95 \%$ and a positive predictive value (PPV) of $80 \%$ [17].

Population data Using Statistics Denmark's databank, we collected data on population size and number of deaths for the County of Funen for the study period in 1 year age and calendar time classes. Place of residence and date of each 
change of residence were collected from the Civil Registration System (CRS) [18] ID in order to account for migration in and out of the county of Funen.

\section{Combination of cohort follow-up and population data}

Using this set-up, information from the database includes all individuals residing in the county of Funen who either had a diagnosis of diabetes and/or underwent amputation during the period 1996-2011. We subdivided follow-up among these by diabetes status (no/yes), amputation status (none/BAA/BKA/AKA), sex, age and period in 1 year intervals, giving a total of 614,318 records. Subtracting the follow-up among individuals with diabetes and/or amputation from that of the total population provided the follow-up from individuals without diabetes and amputation, classified by sex, age and period of follow-up. The total follow-up time classified by diabetes status, amputation status, sex, age and calendar time, as well as the number of diagnoses of diabetes, amputations (BAA, BKA, AKA) and deaths, was also provided from these registrations. In this study, we focus on the transitions to the different amputation states separately among individuals with and without diabetes. The states and transitions between all states are shown in Fig. 1.

\section{Statistical analysis}

Only follow-up from age 15 to 99 years was included in the analysis. We computed directly age-standardised amputation rates by type, year and diabetes status, standardised to the population of individuals without diabetes as of 2011. The dataset used in the analysis consisted of timesplit records from individuals with diabetes or amputation (612,800 records) combined with records of person-years and number of amputation events among non-diabetic individuals with no prior amputation (2,685 records), classified by sex, age and date of follow-up, and duration of diabetes in 1 year steps. We analysed the incidence of the first amputation at each level as well as the incidence of further amputations, subdivided by level of amputation, as illustrated in Fig. 1.

Amputation rates were analysed with proportional hazards Poisson models describing amputation rates as smooth functions of age, calendar time and duration of diabetes (coded as the midpoint of the intervals) using restricted cubic splines. Models also included effect of diabetes and amputation status as well as interaction between sex and diabetes. Estimates of rates and HRs were reported, with $95 \%$ CIs.

All analyses and graphs were made with $\mathrm{R}$ version 3.0.2 [19], using the Epi-package for definition and handling of multistate follow-up [20,21]. A complete account of all data manipulation, statistical analysis and graphical reporting is published as electronic supplementary material (ESM) and is also available at http://bendixcarstensen.com/SDC/Ampu/ FynAmp.pdf

\section{Ethics considerations}

The study is based entirely on register data, and therefore does not require approval from ethics committees according to Danish law. The study was registered with and approved by the Danish Data Protection Agency (Region of Southern Denmark, ID: 2008-58-0035).

\section{Results}

Table 1 shows that the types of amputations are similar in number. Figure 1 provides an overview of the flow of individuals in the study period and shows the rates analysed. The analysis included a total of 2,832 amputations (the coloured arrows in Fig. 1), 1,285 of which were performed in patients with diabetes and 1,547 in individuals without diabetes. Figure 2 shows the directly standardised rates of amputation.

\section{Below-ankle amputation}

We recorded a total of 988 BAAs, of which 483 were among patients with diabetes and 505 among individuals without diabetes. Overall, individuals with diabetes had a 10.7 (95\% CI 9.4, 12.2) fold increased rate of BAA. Table 2 shows that the HR for men was 14.7 (95\% CI $10.5,20.4)$ and for women was 7.5 (95\% CI 5.2, 10.9). Women without diabetes had an incidence of BAA almost half that of men, with an HR of 0.60 (95\% CI 0.51, 0.72). The annual change in amputation for patients with diabetes was $-9.8 \%(95 \% \mathrm{CI}-11.9,-7.6)$. For individuals without diabetes, we found a small insignificant annual change of $-0.5 \%(95 \% \mathrm{CI}-1.4,2.4)$ (Table 3$)$.

\section{From-ankle-to-knee amputation}

We recorded a total of 884 BKAs, 442 of which were performed in individuals with diabetes and 442 in persons without diabetes. Overall, individuals with diabetes had a 7.06 $(95 \%$ CI $6.03,8.27)$ fold increased rate of BKA. Table 2 shows that the HR for men was 7.6 (95\% CI 5.2, 11.3) and for women was 8.4 (95\% CI 5.5, 12.8). Women without diabetes had an incidence of BKA half that of men, with an HR of 0.53 (95\% CI $0.46,0.61)$. The annual change in amputation for patients with diabetes was $-15.1 \%(95 \% \mathrm{CI}-17.6,-12.5)$. For individuals without diabetes, we found a small annual change of $-2.3 \%(95 \% \mathrm{CI}-4.4,-0.2)$ (Table 3$)$. 


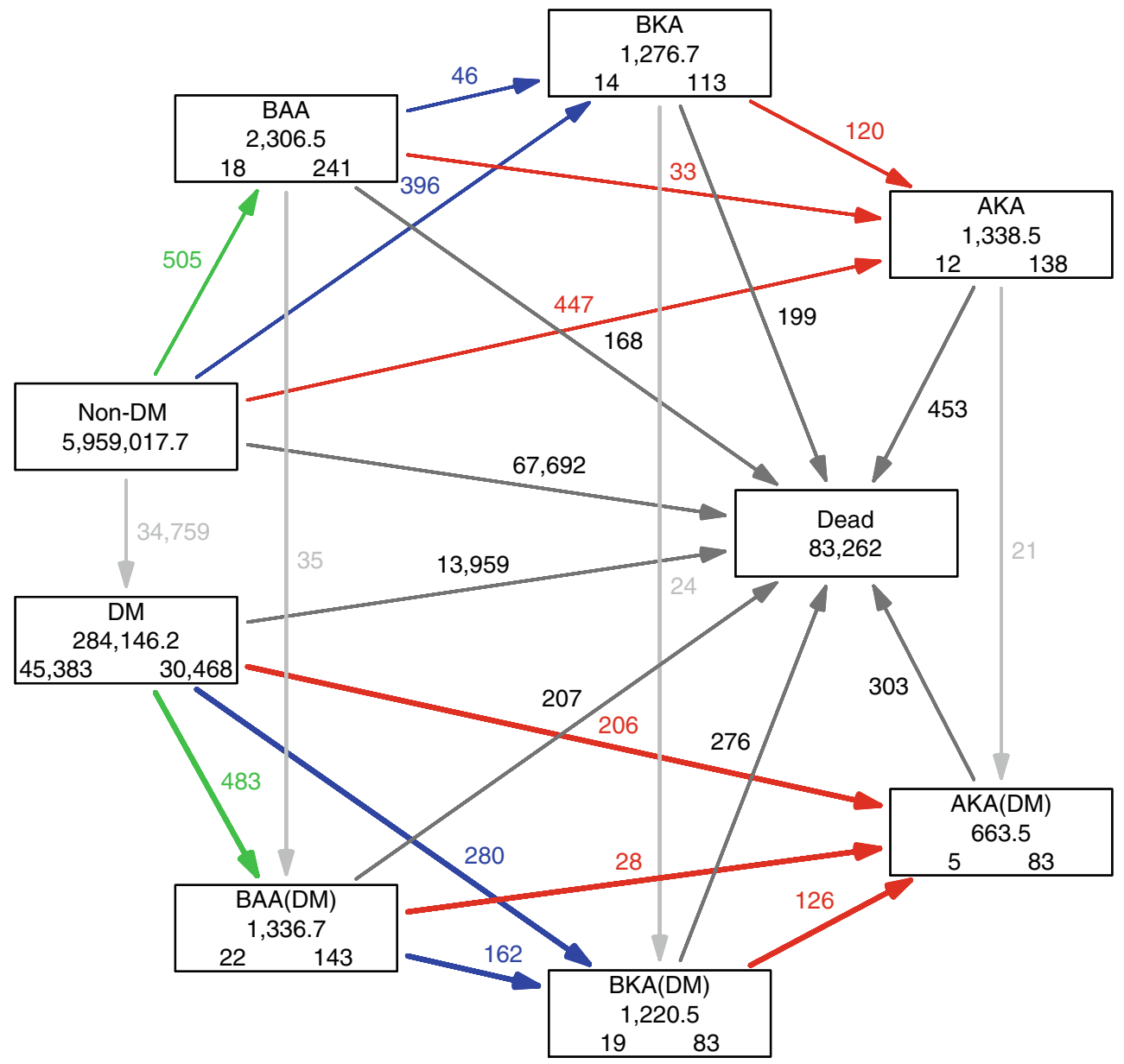

Fig. 1 Patient flow in the study. The current status of individuals with respect to diabetes and/or amputation is shown in the boxes, and the events (diagnosis of diabetes mellitus [DM], amputation or death) are illustrated by the arrows. The same individual can contribute to several boxes according to his or her history of diabetes and amputation. The numbers in the boxes are the number of person-years (centre of each box) and the number of individuals starting (lower left in the box) and ending (lower right in the box) their follow-up in each state. The numbers alongside the arrows (with the same colour as the respective arrow) are the number of observed transitions. The 'Non-DM' state shows only the person-years as this state represents the non-affected part of the Funen population. The analysis in this study concerns the comparison of the thick coloured arrows with their corresponding thin arrows, namely a comparison of the incidence of amputation and amputation level between individuals with and without diabetes. Green arrows, BAA; blue arrows, BKA; red arrows, AKA

Table 1 Number of amputations and person-years with respect to type of amputation and sex

\begin{tabular}{|c|c|c|c|c|c|c|c|c|c|c|c|c|c|c|c|}
\hline \multirow{2}{*}{$\begin{array}{l}\text { DM/amputation } \\
\text { status }\end{array}$} & \multicolumn{5}{|l|}{ Men } & \multicolumn{5}{|c|}{ Women } & \multicolumn{5}{|c|}{ Men and women } \\
\hline & BAA & BKA & AKA & Sum & Person-years & BAA & BKA & AKA & Sum & Person-years & BAA & BKA & AKA & Sum & Person-years \\
\hline Non-DM & 293 & 247 & 209 & 749 & $2,919,600.5$ & 212 & 149 & 238 & 599 & $3,039,417.2$ & 505 & 396 & 447 & 1,348 & $5,959,017.7$ \\
\hline $\mathrm{DM}$ & 351 & 170 & 101 & 622 & $143,239.9$ & 132 & 110 & 105 & 347 & $140,906.4$ & 483 & 280 & 206 & 969 & $284,146.2$ \\
\hline BAA & - & 30 & 18 & 48 & $1,349.5$ & - & 16 & 15 & 31 & 957.0 & - & 46 & 33 & 79 & $2,306.5$ \\
\hline BAA (DM) & - & 122 & 15 & 137 & 988.6 & - & 40 & 13 & 53 & 348.1 & - & 162 & 28 & 190 & $1,336.7$ \\
\hline BKA & - & - & 73 & 73 & 863.8 & - & - & 47 & 47 & 412.9 & - & - & 120 & 120 & $1,276.7$ \\
\hline BKA (DM) & - & - & 77 & 77 & 817.4 & - & - & 49 & 49 & 403.0 & - & - & 126 & 126 & $1,220.5$ \\
\hline All non-DM & 293 & 277 & 300 & 870 & $2,921,813.8$ & 212 & 165 & 300 & 677 & $3,040,787.1$ & 505 & 442 & 600 & 1,547 & $5,962,600.9$ \\
\hline All DM & 351 & 292 & 193 & 836 & $145,045.9$ & 132 & 150 & 167 & 449 & $141,657.5$ & 483 & 442 & 360 & 1,285 & $286,703.4$ \\
\hline Total & 644 & 569 & 493 & 1,706 & $3,066,859.7$ & 344 & 315 & 467 & 1,126 & $3,182,444.7$ & 988 & 884 & 960 & 2,832 & $6,249,304.3$ \\
\hline
\end{tabular}

DM, diabetes mellitus 


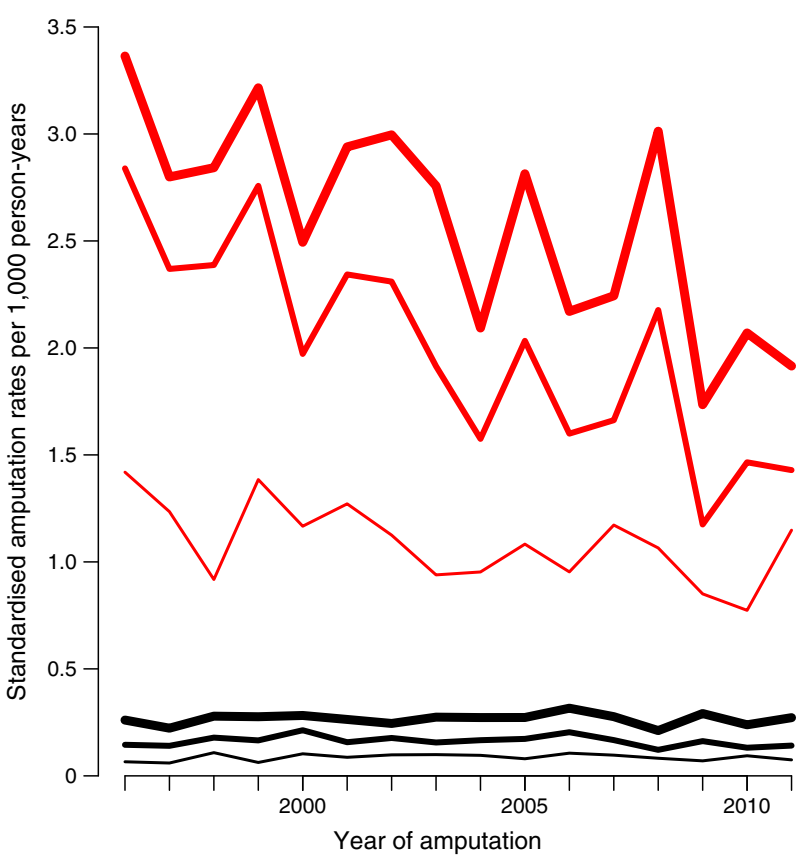

Fig. 2 Age-standardised amputation rates for each year, standardised to the age distribution of individuals without diabetes in the population in 2011. Black curves, individuals without diabetes; red curves, individuals with diabetes. Thin lines, BAA amputations; medium lines, BAA + BKA amputations; thick lines, all amputations (BAA + BKA + AKA)

\section{Above-knee amputation}

We recorded a total of 960 AKAs, of which 360 were among patients with diabetes and 600 among people without diabetes. Overall, individuals with diabetes had a 4.03fold $(95 \%$ CI 3.4, 4.8) increased rate of AKA. Table 2 shows that the HR for men was 4.0 (95\% CI 2.3, 6.7) and for women was 3.7 (95\% CI 2.2, 6.2). Women without diabetes had a 14\% lower incidence than men, with an HR of 0.86 (95\% CI $0.71,1.03)$.

The annual change in amputation for patients with diabetes was insignificant, amounting to $-2.6 \%(95 \% \mathrm{CI}-5.9,0.8)$. For individuals without diabetes, we found a small insignificant annual change of $1.7 \%(95 \% \mathrm{CI}-0.4,3.7)$ (Table 3$)$.

Table 2 Estimated HRs of amputation between individuals with and without diabetes, with no prior amputation, with $95 \% \mathrm{CI}$

\begin{tabular}{|c|c|c|c|c|c|c|}
\hline \multirow[t]{3}{*}{ Sex } & \multicolumn{6}{|c|}{ Type of amputation } \\
\hline & \multicolumn{2}{|l|}{ BAA } & \multicolumn{2}{|c|}{ BKA } & \multicolumn{2}{|c|}{ AKA } \\
\hline & HR & $95 \% \mathrm{CI}$ & HR & $95 \% \mathrm{CI}$ & HR & $95 \% \mathrm{CI}$ \\
\hline Men & 14.7 & $10.5,20.4$ & 7.7 & $5.2,11.3$ & 4.0 & $2.3,6.7$ \\
\hline Women & 7.5 & $5.2,10.9$ & 8.4 & $5.5,12.8$ & 3.7 & $2.2,6.2$ \\
\hline
\end{tabular}

Models were controlled for age, duration of diabetes and prior amputation
Table 3 Annual change (in \%) in amputation rates according to diabetes status and amputation type

\begin{tabular}{|c|c|c|c|c|c|c|}
\hline \multirow[t]{3}{*}{ DM status } & \multicolumn{6}{|c|}{ Type of amputation } \\
\hline & \multicolumn{2}{|c|}{ BAA } & \multicolumn{2}{|l|}{ BKA } & \multicolumn{2}{|l|}{ AKA } \\
\hline & HR & $95 \% \mathrm{CI}$ & HR & $95 \% \mathrm{CI}$ & $\mathrm{HR}$ & $95 \% \mathrm{CI}$ \\
\hline Non-DM & 0.5 & $-1.4,2.4$ & -2.3 & $-4.4,-0.2$ & 1.7 & $-0.4,3.7$ \\
\hline $\mathrm{DM}$ & -9.8 & $-11.9,-7.6$ & -15.1 & $-17.6,-12.5$ & -2.6 & $-5.9,0.8$ \\
\hline
\end{tabular}

Data are changes (in \% per year) of HR between persons with and without DM, with $95 \%$ CIs

Models are controlled for sex, (current) age and duration of diabetes mellitus (DM)

\section{Duration of diabetes}

For all three types of amputation, we saw a high rate of amputation immediately after diagnosis of diabetes. Figures 3, 4, 5 show the absolute rates and HRs for diabetes/non-diabetes as a function of age and duration of diabetes for individuals diagnosed at different ages. Furthermore, the figures show that the HR of amputation between individuals with and without diabetes decreased by increasing age/duration of diabetes, regardless of the age at diagnosis of diabetes. The different curves thus start at age of diagnosis and duration of diabetes equal to 0 , so it can be seen that the initial high rates decrease in about 1 year and the major effect is then age related.

\section{Discussion}

Data on first amputation in individuals with and without diabetes for a period of 16 years in the county of Funen are presented. The total number of amputations amounted to 2,832, of which 1,285 were among individuals with diabetes and 1,547 among individuals without diabetes. We found a substantial reduction by calendar time in BAA rates among patients with diabetes of almost $10 \%$ per year. We also observed a reduction in BKA of $15 \%$ annually in patients with diabetes. We noted a clear sex difference in favour of women.

A major strength of our study is the combination of national and local registers. The use of clinically collected data is often hampered by the fact that registration is inhomogeneous, particularly in relation to how patients with ischaemic or severely infected ulcers are handled. An amputation could be performed at a surgical unit with a focus on registering the amputation per se and recording the diagnosis of diabetes with only a low sensitivity. In our set-up, all amputees were identified via the local administrative system and procedure codes. The codes are 
Fig. 3 (a) BAA rates among individuals without diabetes (broken lines) and individuals with diabetes diagnosed precisely at age 40, 50, 60 and 70 years (solid lines). (b) HRs for BAA between individuals with and without diabetes for age at diagnosis of 40, 50, 60 and 70 years, respectively. Blue, men; red, women. Estimates are shown with $95 \% \mathrm{CI}$ and plotted on a logarithmic scale. A complete account of rates among individuals with prior amputations is given in the ESM a

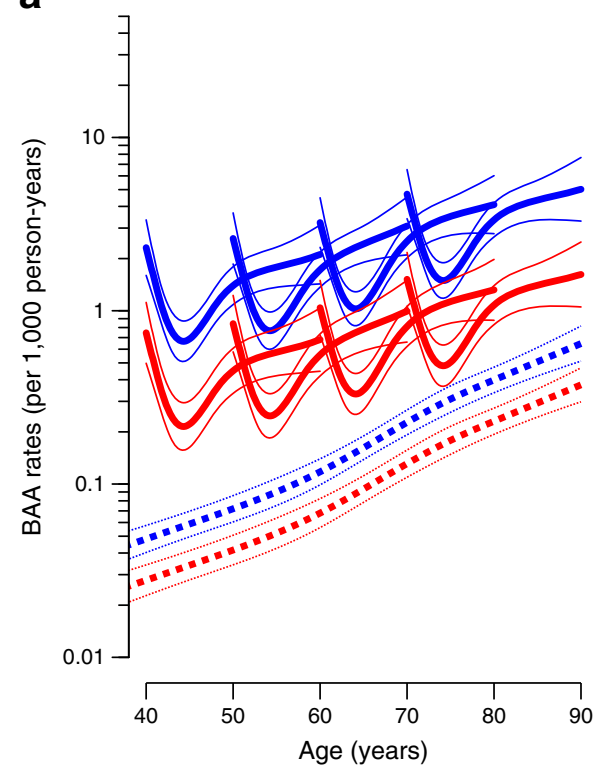

b

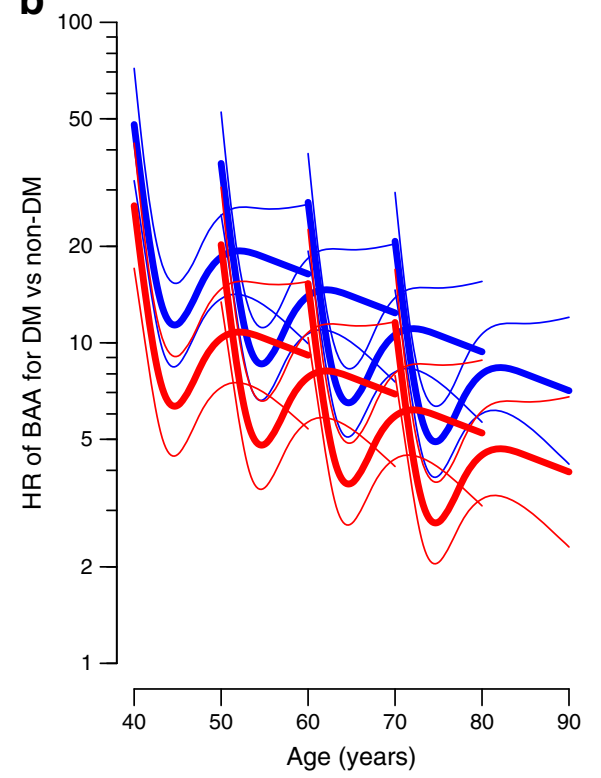

essentially related to diagnosis-related group reimbursement and thus focused on when registration is obtained. These include all types of amputations. Hypothetically, some amputations could have been missed if the amputation was performed in an emergency department. However, this number is expected to be small. We combined data from NDR, CRS and local electronic healthcare platforms for the County of Funen, Denmark to describe the population frequency of amputation among individuals with as well as without diabetes. We trust our approach to be the most reliable way of obtaining valid data collection, since all patients with diabetes and all amputations were included; thus no selection bias was introduced.
One of the study's strengths as well as a limitation is the use of the NDR. A methodological limit with respect to the NDR is the inclusion in it of individuals with diabetes identified not only by diagnosis, but also by a defined criterion; this limitation has been acknowledged by others as well [6]. A recent study by Green et al investigated the NDR and found that the register has a completeness (sensitivity) of $\geq 95 \%$ and an assumed PPV of $80 \%$ or above in the Danish population [17]. This indicates that some of the patients in NDR are falsely included as patients with diabetes. With regard to our study, the impact would be minor inasmuch as the presumed group of
Fig. 4 (a) BKA rates among individuals without diabetes (broken lines) and individuals with diabetes diagnosed precisely at age 40, 50, 60 and 70 years (solid lines). (b) HRs for BKA between individuals with and without diabetes for age at diagnosis of 40, 50, 60 and 70 years, respectively. Blue, men; red, women. Estimates are shown with $95 \% \mathrm{CI}$ and plotted on a logarithmic scale. A complete account of rates among individuals with prior amputations is given in the ESM a

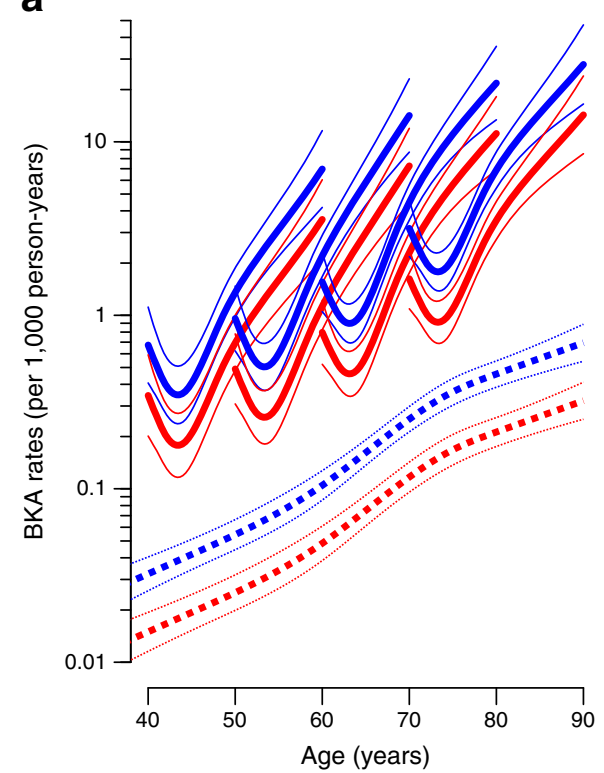

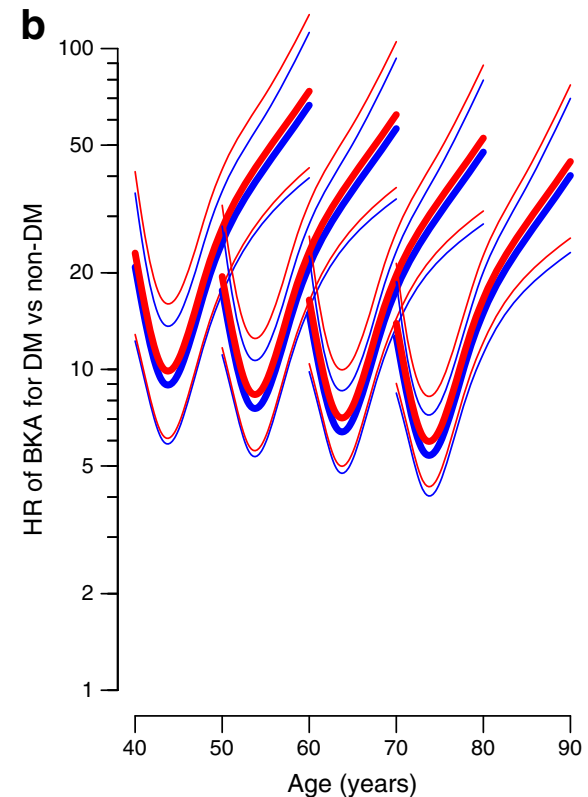


Fig. 5 (a) AKA rates among individuals without diabetes (broken lines) and individuals with diabetes diagnosed precisely at age 40, 50, 60 and 70 (solid lines). (b) HRs for AKA between individuals with and without diabetes for ages at diagnosis of 40, 50, 60 and 70 years, respectively. Blue, men; red, women. The HR was independent of sex; hence the black curves refer to both men and women. Estimates are shown with $95 \% \mathrm{CI}$ and plotted on a logarithmic scale. A complete account of rates among individuals with prior amputations is given in the ESM a

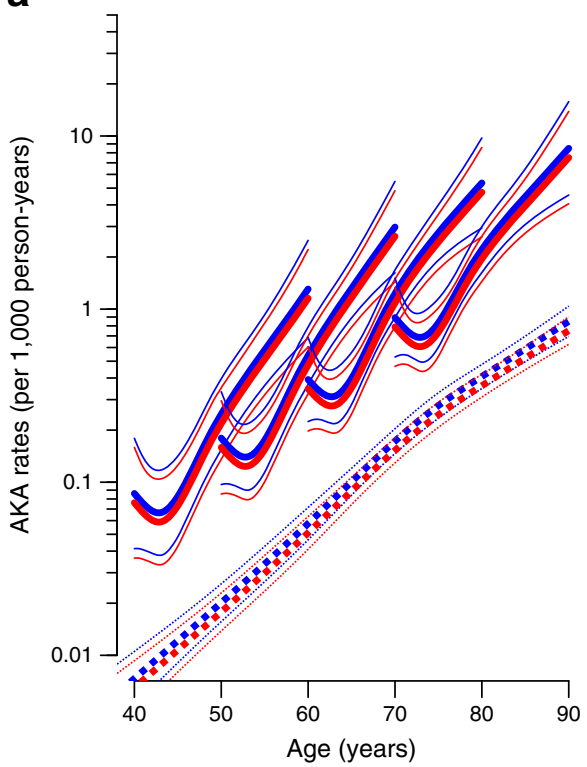

b

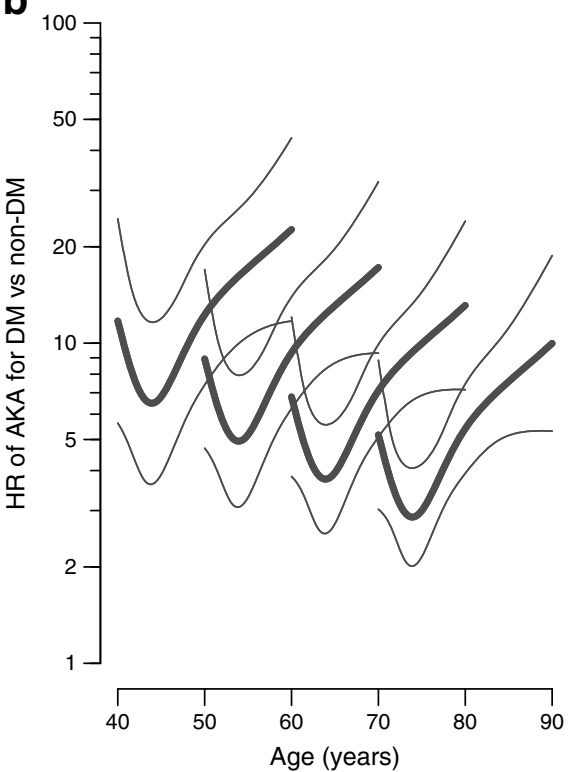

falsely registered diabetes patients included young women with polycystic ovarian syndrome and gestational diabetes.

A recent Danish study by Jorgensen et al showed a reduction in the rate of major, but not minor, amputations [7]. In contrast to the data presented here, individuals with type 2 diabetes in their study population were highly selected and thus not representative of the general population. Data for our study were collected from the county of Funen without selection bias. The data presented here constitute a valid subsample of the Danish population that could reflect the general trend in Denmark. Data from these two studies, however, show the same encouraging trend of a reduction in the rate of amputations, not only major amputations (BKA + AKA), but also minor ones (BAA).

Although many different methodological approaches have been undertaken [8], the results presented here are substantiated by many other studies $[6,12]$ showing a global reduction in the relative number of amputations among patients with diabetes. Furthermore, numerous studies have shown that individuals suffering from diabetes have a several-fold increased risk of amputation [8, 22].

During the observation period, the 'Funen model' for diabetes care was implemented. This includes regular foot inspection, better self-care and the establishment of specialised wound clinics in the uptake area consisting of a multidisciplinary wound care team. Evidence is pointing towards better wound healing using this approach [23]. The set-up leads to stratification of patients with diabetes and then construction of individual schedules to obtain the best possible care. Our study suggests that the reduction in amputation rates among patients with diabetes is most likely to be due to improvements in the care of individuals with diabetes and not to a general improvement in the treatment of patients with ischaemia or neuropathy, since the amputation rate in nondiabetic individuals was unchanged. The introduction of vascular surgery, improved surgical techniques and empirically based antibiotic regimens cannot explain our findings, since these procedures are applied equally in individuals with and without diabetes. The findings in individuals with diabetes can therefore only be explained by improved diabetes care, for example, improved metabolic control or changes in organisation; we believe it to be due to both. Screening procedures for diabetes may have implications with respect to earlier and more aggressive treatment leading to fewer complications, as demonstrated by the legacy effect found from the UK Prospective Diabetes Study [24].

The decline in amputation rates has been suggested to be a consequence of introducing vascular surgery [10]. However, because we saw no decline in amputation rates among nondiabetic individuals, the decline in amputation rate among patients with diabetes cannot be explained by a general improvement in amputation prevention, such as easier access to vascular surgery. This observation has been substantiated by others [12]. It does not, however, rule out the effect of vascular surgery as an important factor for the reduction in number of amputations. Other factors with potential implications for the reduction in critical limb ischaemia and major amputations among patients with diabetes include higher adherence to statin therapy $[25,26]$ and other treatment strategies, including a number of interventions that may affect the amputation rate [27].

In this study, we investigated amputations at three levels (low [BAA], mid [BKA] and high [AKA]). This was done in order to distinguish outcome in terms of a more detailed level of amputation. In most studies, amputation is only defined as minor (BAA) or major (BKA + AKA), although other 
definitions have been used [12]. We found a substantial reduction by calendar time in BAA and BKA, but no significant reduction in AKA among individuals with diabetes. Others argue that minor (BAA) amputations are often undertaken in an attempt to avoid major (BKA and AKA) amputations [7]. This might be the case in the County of Funen as well, which translates into an annual reduction for BAA of $10 \%$, but even higher for BKA, amounting to $15 \%$. We observed only a small change in AKA. This may indicate that the general improvement in the care of patients with diabetes may not have affected the rate of AKA. Others have shown a general reduction in amputations using a two-level definition of amputation compared with three as presented here [28].

Despite the weaknesses outlined above, this material is unique and highly valid. The results are encouraging as they show a general reduction in the number of amputations performed in diabetic patients. A higher risk of amputation in individuals with diabetes, especially among men compared with women, is substantiated in other studies [29] and deserves attention. A shared-care model is necessary in which general practitioners are offered a screening tool to secure a regular outpatient clinical attendance and collaborative effort with the specialists at the hospital (e.g. the Funen model). Furthermore, it is essential to have data registration linked to national databases so that medical treatment can be optimised. Initiatives like these are necessary to achieve further reductions in the occurrence of complications linked to diabetes.

\section{Conclusions}

The reduction in amputations among diabetic individuals is encouraging. The results presented here indicate that it is beneficial to adopt a multidisciplinary approached optimised for screening and treating complications linked to diabetes. It is also encouraging that the overall amputation rate is declining in most parts of the world. However, amputation rates in patients with diabetes remain high compared with individuals without diabetes, posing a great challenge for improving care. We confirm a sex difference, with a higher amputation rate among men. We believe the data presented here constitute the best available extracts from well-validated registers and could serve as a model for obtaining correct data sampling in future studies.

Acknowledgements We are grateful to D. F. G. Jensen, Department for Budget and Data, Odense University Hospital, Denmark, for help with data access and handling of the register data.

Funding This research received no specific grant from any funding agency in the public, commercial or not-for-profit sectors.

Duality of interest The authors declare that there is no duality of interest associated with this manuscript.
Contribution statement $\mathrm{BSBR}, \mathrm{BC}, \mathrm{HBN}, \mathrm{OS}$ and $\mathrm{KBY}$ conceived and designed the study. $\mathrm{BC}$ and BSBR undertook the data-handling. $\mathrm{BC}$ analysed the data. BSBR, KBY and BC wrote the paper. Manuscript review was undertaken by $\mathrm{BC}, \mathrm{HBN}, \mathrm{OS}, \mathrm{KBY}$ and $\mathrm{BSBR}$. All authors read, revised and approved the final version of the manuscript. HBN is the guarantor of this work.

\section{References}

1. Brownrigg JR, Davey J, Holt PJ et al (2012) The association of ulceration of the foot with cardiovascular and all-cause mortality in patients with diabetes: a meta-analysis. Diabetologia 55:29062912

2. Apelqvist J, Larsson $\mathrm{J}$ (2000) What is the most effective way to reduce incidence of amputation in the diabetic foot? Diabetes Metab Res Rev 16(Suppl 1):S75-S83

3. Apelqvist J, Ragnarson-Tennvall G, Larsson J, Persson U (1995) Long-term costs for foot ulcers in diabetic patients in a multidisciplinary setting. Foot Ankle Int 16:388-394

4. Kristensen MT, Holm G, Kirketerp-Moller K, Krasheninnikoff M, Gebuhr P (2012) Very low survival rates after non-traumatic lower limb amputation in a consecutive series: what to do? Interact Cardiovasc Thorac Surg 14:543-547

5. Holman N, Young RJ, Jeffcoate WJ (2012) Variation in the recorded incidence of amputation of the lower limb in England. Diabetologia 55:1919-1925

6. Ikonen TS, Sund R, Venermo M, Winell K (2010) Fewer major amputations among individuals with diabetes in Finland in 1997 2007: a population-based study. Diabetes Care 33:2598-2603

7. Jorgensen ME, Almdal TP, Faerch K (2014) Reduced incidence of lower-extremity amputations in a Danish diabetes population from 2000 to 2011. Diabet Med 31:443-447

8. Moxey PW, Gogalniceanu P, Hinchliffe RJ et al (2011) Lower extremity amputations - a review of global variability in incidence. Diabet Med 28:1144-1153

9. Trautner C, Haastert B, Mauckner P, Gatcke LM, Giani G (2007) Reduced incidence of lower-limb amputations in the diabetic population of a German city, 1990-2005: results of the Leverkusen Amputation Reduction Study (LARS). Diabetes Care 30:26332637

10. Holstein P, Ellitsgaard N, Olsen BB, Ellitsgaard V (2000) Decreasing incidence of major amputations in people with diabetes. Diabetologia 43:844-847

11. Armstrong DG, Bharara M, White M et al (2012) The impact and outcomes of establishing an integrated interdisciplinary surgical team to care for the diabetic foot. Diabetes Metab Res Rev 28: 514-518

12. Witso E, Lium A, Lydersen S (2010) Lower limb amputations in Trondheim, Norway. Acta Orthop 81:737-744

13. Gottrup F, Holstein P, Jorgensen B, Lohmann M, Karlsmar T (2001) A new concept of a multidisciplinary wound healing center and a national expert function of wound healing. Arch Surg 136: $765-772$

14. Krishnan S, Nash F, Baker N, Fowler D, Rayman G (2008) Reduction in diabetic amputations over 11 years in a defined U.K. population: benefits of multidisciplinary team work and continuous prospective audit. Diabetes Care 31:99-101

15. Bay-Nielsen H (2011) NOMESCO Classification of Surgical Procedures (NCSP). Available from http://nowbase.org/ /media/ Projekt $\% 20$ sites/Nowbase/Publikationer/NCSP/NCSP\%201_16. ashx, accessed 4 Jun 2015

16. Carstensen B, Kristensen JK, Ottosen P, Borch-Johnsen K, Steering Group of the National Diabetes R (2008) The Danish National 
Diabetes Register: trends in incidence, prevalence and mortality. Diabetologia 51:2187-2196

17. Green A, Sortso C, Jensen PB, Emneus M (2015) Validation of the Danish National Diabetes Register. Clin Epidemiol 7:5-15

18. Pedersen CB (2011) The Danish Civil Registration System. Scand J Public Health 39:22-25

19. R Core Team (2015) A language and environment for statistical computing. R Foundation for Statistical Computing, Vienna, Austria. Available from https://www.R-project.org/, accessed 4 Jun 2015

20. Plummer M, Carstensen B (2011) Lexis: an R class for epidemiological studies with long-term follow-up. J Stat Softw 38:1-12

21. Carstensen B, Plummer M (2011) Using Lexis objects for multistate models in R. J Stat Softw 38:1-18

22. Pscherer S, Dippel FW, Lauterbach S, Kostev K (2012) Amputation rate and risk factors in type 2 patients with diabetic foot syndrome under real-life conditions in Germany. Prim Care Diabetes 6:241246

23. Gottrup F (2005) Management of the diabetic foot: surgical and organisational aspects. Horm Metab Res 37(Suppl 1):69-75

24. Chalmers J, Cooper ME (2008) UKPDS and the legacy effect. N Engl J Med 359:1618-1620
25. Suckow BD, Kraiss LW, Schanzer A et al (2014) Statin therapy after infrainguinal bypass surgery for critical limb ischemia is associated with improved 5-year survival. J Vasc Surg 61:126-133

26. Westin GG, Armstrong EJ, Bang H et al (2014) Association between statin medications and mortality, major adverse cardiovascular event, and amputation-free survival in patients with critical limb ischemia. J Am Coll Cardiol 63:682-690

27. Malik RA, Tesfaye S, Ziegler D (2013) Medical strategies to reduce amputation in patients with type 2 diabetes. Diabet Med 30:893900

28. Tseng CL, Rajan M, Miller DR, Lafrance JP, Pogach L (2011) Trends in initial lower extremity amputation rates among Veterans Health Administration Health Care System users from 2000 to 2004. Diabetes Care 34:1157-1163

29. Alvarsson A, Sandgren B, Wendel C, Alvarsson M, Brismar K (2012) A retrospective analysis of amputation rates in diabetic patients: can lower extremity amputations be further prevented? Cardiovasc Diabetol 11:18 